\title{
DRIP IRRIGATION MANAGEMENT SYSTEM AND MONITORING SOIL USING WIRELESS SENSORS
}

\author{
Jayashree Shiral $^{1 *}$, Monika Hazra ${ }^{2}$, Prajakta Thakre ${ }^{3}$, Ankita Gawande ${ }^{4}$, Karishma Bhadang $^{5}$ \\ *1,2,3,4,5 DBACER, Nagpur \\ ${ }^{2}$ Email: immonika.hazra@gmail.comswtMaithu@gmail.com
}

*Corresponding Author: -

\begin{abstract}
: -
In this paper, we are using two sensors to sense the humidity and temperature of the soil. water is very precious to all human being as well as to plants. With the help of drip irrigation water will be maintained at constant level which means the water will reach to the roots drop by drop if the field is irrigated heavily with a water, there are chances that a plant may die because of unnecessary irrigation. The water could also wash them away during irrigation if very strong force of water is released at the same time. On the other hand, if there is not enough water, then also there may be chances that the plants may die due to lack of water. With the help of atomized drip irrigation system, the sensor will detect the need of water by sensing the temperature \& moisture and inform to the control field programmable gate array (FPGA) which will on the motor and provide water to the needed field area. Due to which the healthy plants will grow which leads to increase the productivity.
\end{abstract}

Keywords: -Drip Irrigation, Sensors, FPGA (Field programmable gate array), Temperature sensor, Humidity sensor. 


\section{INTRODUCTION}

The Irrigation is the artificial application of water to the soil for assisting in growing crops. Drip irrigation also known as micro irrigation \& trickle is an irrigation method which minimizes the use of water \& fertilizer by providing water drop by drop to the plants and onto the soil surface or directly onto the root zone due to which a large quantity of water is saved $\&$ it is use in another work also the fertilizer which comes to the plant with the water. Indian agriculture is dependent on the seasons which is dependent on the nature and unreliable source of water, water is very important for the plants growth, so there is a need for an automatic irrigation system in the country which can reduce the water and provide water to the farms according to their requirements. For a big farm land with horticulture activity the solution will be an automated system. Water in the soil is called soil moisture and it is very important for plant growth. The advantage of this system using wireless sensors is to control all related parameters for better irrigation management. This paper gives a review of remote monitoring and control system based on existing technologies i.e, FPGA based remote control and monitoring system with automatic irrigation management is proposed in this project.

\subsection{Methodology}

There are important parameters to be measured for automation in irrigation system is soil moisture and temperature. The whole field is divided into two regions such that each region should contain one moisture sensor and a temperature sensor. DS1621 can be used as a temperature sensor to detect temperature while DHT11 can be used as the moisture sensor to detect moisture. These sensors are covered into the ground at required intensity. Once the soil has reached desired moisture level the sensors send a message to the controller to turn on motor to flow down the water. The message send by the sensor is boosted up to the required level by corresponding amplifier stages.

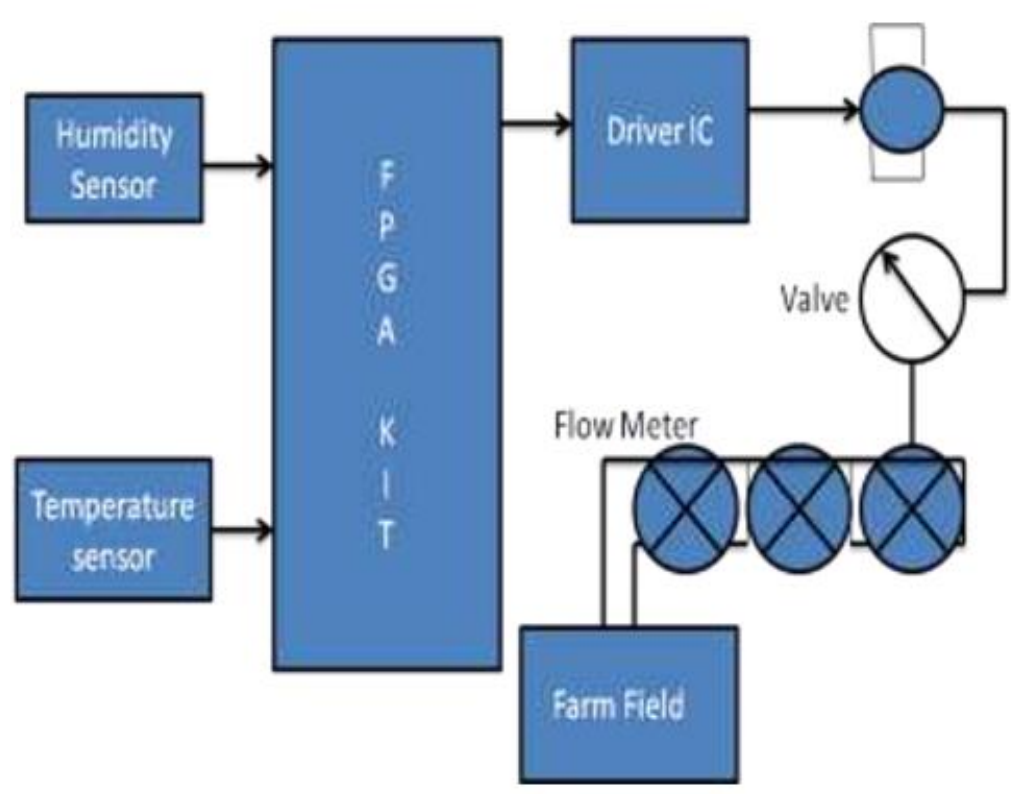

Fig 1. System Architecture

There is an LCD module can be used in the system to monitor current readings of all the sensors. While applying the automation on large fields more than one such sensors can be interfaced with the Centralized system i.e. FPGA kit \& the microcontroller is inbuilt in FPGA kit. microcontroller unit may warn the insufficient amount of water input with the help of flowmeter.

\subsection{SENSORS}

\subsubsection{DS1621 Temperature Sensor Features}

- For measuring temperature no external component is required

- Temperature is read as a 9-bit value (2-byte transfer) Wide power supply range (2.7V to 5.5V)

- Temperature would be converted to digital form in less than one second.

- Thermostate output signal settings are user definable and nonvolatile.

\subsubsection{DHT11 Humidity Sensor Features}

- Low cost, stable for long-term.

- Humidity and Temperature should be measured reletively.

- Excellent quality and fast response.

- Long distance signal transmission.

- Digital signal output and exact calibration. 


\subsection{L293D Driver Ic}

- L293D is a typical motor driver IC which allows DC motor to drive on both directions.

- L293D is a 16-pin IC which controls a set of two DC motors in any direction simultaneously.

- This means that you can control two DC motor driver integrated circuit (IC).

\subsection{XILINX CPLD FEATURE}

- It is a fast concurrent programming.

- It's control on individual outputs.

- It enhances the data security.

- It provides high quality and reliability

- Pin-compatible with 5V-core XC9572 device in the 44-pin PLCC package and the 100-pin TQFP

\section{Literature Survey}

After the research in the agricultural field, researchers founded that the field of agriculture goes on declining day by day. Use of technology in the field of agriculture plays important role in raising the production as well as in reducing the extra man power efforts. Some of the researchers tried for betterment of farmers and provides the systems that use technologies which are helpful for raising the agricultural field. Some of the researchers approved out in field of agriculture are summarized here.

[A] Aniket H. Hade, Dr. M.K. Sengupt,whose title of the project was 'Automatic control of drip irrigation system \& monitoring of soil by wireless' introduced, A various drip irrigation methods have been proposed but they have been found to be very comfortable and obscure to use. The farmer has to observe on irrigation schedule in the conventional drip irrigation system which is different in diverse types of crops. There future scope was that paper will try to apply food nutrients to the plant and crop by air mixed with very less water with better monitoring and processing in Apr. 2014. [B] Mr. S.G. Galande, Dr. G.H. Agrawal whose title of the project was 'Embedded Controlled Drip Irrigation System, introduced, the conventional irrigation methods like flood type feeding systems usually wet the lower leaves and stem of the plants. The entire soil surface is flooded and often stays wet long after irrigation is completed and their future scope will introduce different kinds of sensors that is, temperature, humidity, and etc. September - October 2013.

\section{Proposed System}

There are various factors for the betterment of agriculture depends on various environmental parameters such as temperature, soil moisture, relative humidity, $\mathrm{pH}$ of soil etc. Changes in any of these parameters can cause problems like diseases, improper growth of plant, etc. which resulting in lesser yield. Block diagram of the proposed system shown in Fig 2. Consists of different types of sensors such as temperature sensor and humidity sensor.

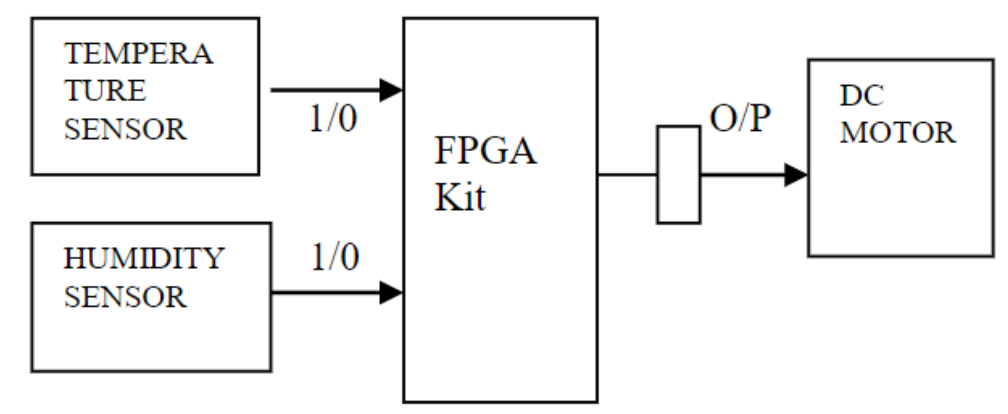

Fig 2. Basic block diagram of proposed system

In which when the temperature set is equal to or greater than the threshold value then it will pass the message to the controller and then the DC motor will start. The rotation of the DC motor is depending on the set input of user weather to rotate the motor in clock wise direction or anti-clock. According to which water will start flowing through pipes drop by drop and goes into the root zone. After the condition is been satisfied the motor will be stop rotating.

\section{Flowchart}

Fig. 3 shows the flowchart of the system. First block is the initialization of the system and then it will sense the temperature and moisture of the soil. It reads the parameter, if the soil moisture contain is less than the threshold limit then it indicates requirement of water for plants. And this process will repeat until the temperature and moisture of the soil is less than the set limit. 


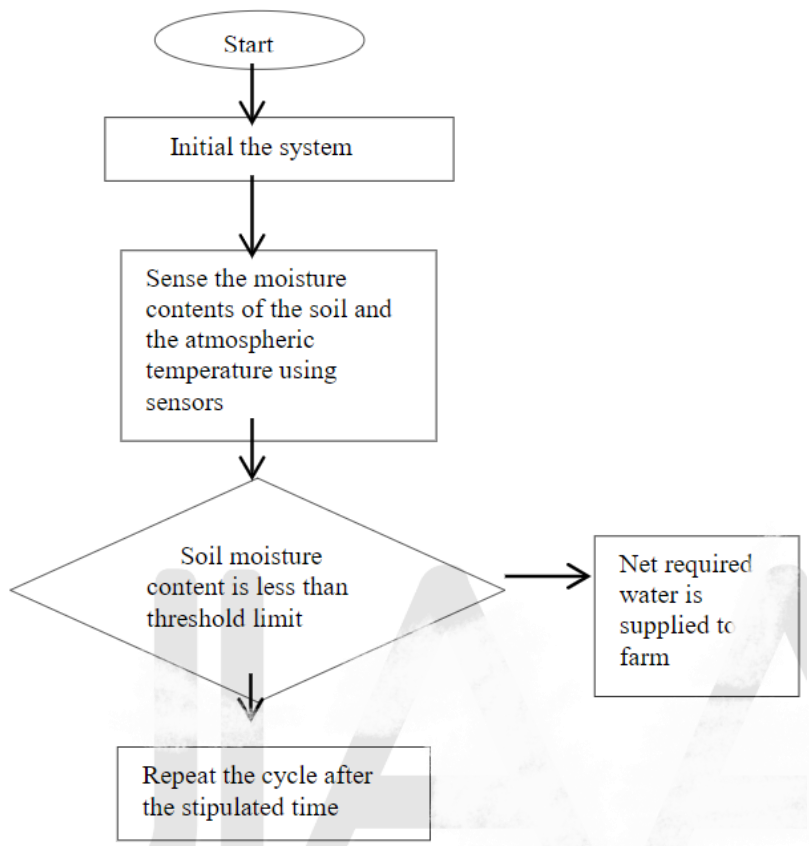

Fig.3 Flowchart

\section{Conclusion}

This technique proves to be a real time feedback control system which monitors and controls all the activities of drip irrigation system efficiently. It helps farmer to get more productivity with less investment. Using this system, less manpower is required. Excess amount of water will not be consumed by the soil.

\section{References}

[1].Er.Sukhjit Singh, Er.Neha Sharma, "Research Paper on Drip Irrigation Management using wireless sensors", ISSN: 2250-3501Vol.2, No4, August 2012.

[2].Aniket H. Hade, Dr. M.K. Sengupta," Automatic control of drip irrigation system \& monitoring of soil by wireless",Volume 7, Issue 4 Ver. III PP 57-61, Apr. 2014.

[3].Mr. S.G. Galande, Dr. G.H. Agrawal," Embedded Controlled Drip Irrigation System”, Volume 2, Issue 5, September - October 2013 Conference on Agricultural Engineering (AgEng2008). Conf. Proc. p.19.

[4].Callaway, E.H. "Wireless sensor networks: architectures and protocols". Auerbach Publications: New York, NY, USA, 2004.

[5].Qingshan, S.; Ying, L.; Gareth, D.; Brown, D."Wireless intelligent sensor networks for refrigerated vehicle". In IEEE 6th Symposium on Emerging Technologies Mobile and Wireless Communication, Shanghai, China, 2004.

[6].Mahir Dursun and Semih Ozden, "A wireless application of drip irrigation automation supported by soil moisture sensors", Scientific Research and Essays Vol. 6(7), pp. 1573-1582, 4 April, 2011.

[7].Baggio A (2005). "Wireless sensor networks in precision agriculture". In: On-line Proc. of the Workshop on RealWorld Wireless Sensor Networks, pp. 50-51.

[8].Vories, E.D., Glover, R.E., Bryant, K.J., Tacker, P.L., 2003. Estimating the cost of delaying irrigation for mid- south cotton on clay soil.In: Proceedings of the 2003 Beltwide Cotton Conference National Cotton Council, Memphis, TN, USA, pp. 656-661.

[9]. Mahesh M. Galgalikar, Gayatri S Deshmukh "Real- Time Automization of Irrigation system for Social Modernization of Indian Agricultural System” 2010

[10]. Abreu VM, Pereira LS (2002). Sprinkler irrigation systems design using ISAMim. p. 022254. Baggio A (2005).

[11]. Wireless sensor networks in precision agriculture. In: On-line Proc, of the Workshop on Real-World Wireless Sensor Networks, pp. 50-51.

[12]. Balendonck J, Hemming J, Van Tuijl BAJ, Pardossi A, Incrocci L, Marzialetti P (2008). Sensors and Wireless Sensor Networks for Irrigation Management under Deficit Conditions (FLOW-AID). International

[13]. Camilli A, Cugnasca CE, Saraiva AM, Hirakawa AR, Corrêa LP (2007). From wireless sensor to field mapping: Anatomy of an application for precision agriculture. Comput. Electron. Agric., 58: 25-36

[14]. Coates RW, Delwiche M, Brown P (2005). Precision Irrigation in Orchards: Development of a Spatially Variable Micro Sprinkler System. Information and Technology for Sustainable Fruit and Vegetable Production (FRUTIC). pp. 611-624.

[15]. Coates RW, Delwiche MJ (2006). Solar-Powered, Wirelessly-Networked Valves for Site- Specific Irrigation. ASABE Annual International Meeting. Paper, pp. 062165

[16]. Coates RW, Delwiche MJ, Brown PH (2006a). Control of individual micro sprinklers and fault detection strategies. Precision Agric.,7: 85-99.

[17]. Coates RW, Delwiche MJ, Brown PH (2006b). Design of a system for individual micro sprinkler control. Trans. ASABE, 49(6): 1963-1970. 